\title{
Dynamic Wordclouds and Vennclouds for Exploratory Data Analysis
}

\author{
Glen Coppersmith \\ Human Language Technology Center of Excellence \\ Johns Hopkins University \\ coppersmith@ jhu.edu
}

\author{
Erin Kelly \\ Department of Defense \\ elkelly8@gmail.com
}

\begin{abstract}
The wordcloud is a ubiquitous visualization of human language, though it falls short when used for exploratory data analysis. To address some of these shortcomings, we give the viewer explicit control over the creation of the wordcloud, allowing them to interact with it in real timea dynamic wordcloud. This allows iterative adaptation of the visualization to the data and inference task at hand. We next present a principled approach to visualization which highlights the similarities and differences between two sets of documents - a Venncloud. We make all the visualization code (primarily JavaScript) freely available.
\end{abstract}

\section{Introduction}

A cornerstone of exploratory data analysis is visualization. Tremendous academic effort and engineering expertise has created and refined a myriad of visualizations available to the data explorer, yet there still exists a paucity of options for visualizing language data. While visualizing human language is a broad subject, we apply Polya's dictum, and examine a pair of simpler questions for which we still lack an answer:

\section{- (1) what is in this corpus of documents?}

\section{- (2) what is the relationship between these two corpora of documents?}

We assert that addressing these two questions is a step towards creating visualizations of human language more suitable for exploratory data analysis. In order to create a meaningful visualization, one must understand the inference question the visualization is meant to inform (i.e., the reason for which (1) is being asked), so the appropriate aspects of the data can be highlighted with the aesthetics of the visualization. Different inference questions require different aspects to be highlighted, so we aim to create a maximally-flexible, yet simple and intuitive method to enable a user to explore the relevant aspects of their data, and adapt the visualization to their task at hand.

The primary contributions of this paper are:

- A visualization of language data tailored for exploratory data analysis, designed to examine a single corpus (the dynamic wordcloud) and to compare two corpora (the Venncloud);

- The framing and analysis of the problem in terms of the existing psychophysical literature;

- Distributable JavaScript code, designed to be simple to use, adapt, and extend.

We base our visualizations on the wordcloud, which we deconstruct and analyze in $\S 3$ and $\S 4$. We then discuss the literature on wordclouds and relevant psychophysical findings in $\S 5$, taking guidance from the practical and theoretical foundations explored there. We then draw heavily on similarities to more common and well understood visualizations to create a more useful version of the wordcloud. Question (1) is addressed in $\S 7$, and with only a small further expansion described in $\S 8$, an approach to (2) becomes evident.

\section{Motivating Inference Tasks}

Exploratory data analysis on human language encompasses a diverse set of language and inference tasks, so we select the following subset for their variety. One task in line with question (1) is getting the general subject of a corpus, highlighting content-bearing words. One might want to examine a collection of social media missives, too numerous to read individually, perhaps to detect emerging news (Petrovic et al., 2013). Separately, author identification (or idiolect analysis) 
attempts attribution of documents (e.g., Shakespeare's plays or the Federalist papers) by comparing the author's writing style, focusing on stylistic and contentless words - for a review see (Juola, 2006). Further, some linguistic psychometric analysis depends on the relative distribution of pronouns and other seemingly contentless words (Coppersmith et al., 2014a; Chung and Pennebaker, 2007).

Each of these questions involves some analysis of unigram statistics, but exactly what analysis differs significantly, thus no single wordcloud can display all of them. Any static wordcloud is a single point in a distribution of possible wordclouds - one way of calculating statistics from the underlying language and mapping those calculations to the visual representation. Many such combinations and mappings are available, and the optimal wordcloud, like the optimal plot, is a function of the data and the inference task at hand. Thus, we enable the wordcloud viewer to adjust the relationship between the aspects of the data and the aesthetics of the display, which allows them to view different points in the distribution of possible wordclouds. The dynamic wordcloud was implicitly called for in (Rayson and Garside, 2000) since human expertise (specifically knowledge of broader contexts and common sense) is needed to separate meaningful and non-meaningful differences in wordclouds. We enable this dynamic interaction between human and visualization in realtime with a simple user interface, requiring only a modicum more engineering than the creation of a static wordcloud, though the depth of extra information conveyed is significant.

\section{Wordcloud Aesthetics}

We refer to each visual component of the visualization as an aesthetic (ala (Wickham, 2009)) each aesthetic can convey some information to the viewer. For context, the aesthetics of a scatterplot include the $x$ and $y$ position, color, and size of each point. Some are best suited for ordinal data (e.g., font size), while others for categorical data (e.g., font color).

Ordinal data can be encoded by font size, the most prominent and noticeable to the viewer (Bateman et al., 2008). Likewise, the opacity (transparency) of the word is a prominent and ordinal aesthetic. The order in which words are displayed can convey a significant amount of infor- mation as well, but using order in this fashion generally constrains the use of $x$ and $y$ position.

Categorical data can be encoded by the color of each word - both the foreground of the word itself and the background space that surrounds it (though that bandwidth is severely limited by human perception). Likewise for font weight (boldness) and font decoration (italics and underlines). While font face itself could encode a categorical variable, making comparisons of all the other aspects across font faces is likely to be at best uninformative and at worst misleading.

\section{Data Aspects}

As the wordcloud has visual aesthetics that we can control ( $(3)$, the data we need to model has aspects that we want to represent with those aesthetics. This aspect-to-aesthetic mapping is what makes a useful and informative visualization, and needs to be flexible enough to allow it be used for a range of inference tasks.

For clarity, we define a word $(w)$ as a unique set of characters and a word token (w) as a single usage of a word in a document. We can observe multiple word tokens (w) of the same word $(w)$ in a single document $(d)$. For any document $d$ we represent the term frequency of $w$ as $t f_{d}(w)$. Similarly, the inverse document frequency of $w$ as $i d f(w)$. A combination of $t f$ and $i d f$ is often used to determine important words in a document or corpus. We focus on $t f$ and $i d f$ here, but this is just an example of an ordinal value associated with a word, there are many other such word-ordinal pairings that are worth exploring (e.g., weights in a classifier).

The dynamic range ("scaling" in (Wickham, 2009)) also needs to be considered, since the data has a natural dynamic range - where meaningful differences can be observed (unsurprisingly, the definition of meaningful depends on the inference task). Likewise, each aesthetic has a range of values for which the users can perceive and differentiate (e.g., words in a font size too small are illegible, those too large prevent other words from being displayed; not all differences are perceptible). Mapping the relevant dynamic range of the data to the dynamic range of the visualization is at the heart of a good visualization, but to do this algorithmically for all possible inference tasks remains a challenge. We, instead, enable the user to adjust the dynamic range of the visualization explicitly. 


\section{Prior Art}

Wordclouds have a mixed history, stemming from Jim Flanagan's "Search Referral Zeitgeist", used to display aggregate information about websites linking to his, to its adoption as a visual gimmick, to the paradoxical claim that 'wordclouds work in practice, but not in theory' (see (Viégas and Wattenberg, 2008) for more). A number of wordcloud-generators exist on the web (e.g., (Feinberg, 2013; Davies, 2013)), though these tend towards creating art rather than informative visualizations. The two cited do allow the user limited interaction with some of the visual aesthetics, though not of sufficient scope or response time for general exploratory data analysis.

Enumerating all possible inference tasks involving the visualization of natural language is impossible, but the prior art does provide empirical data for some relevant tasks. This further stresses the importance of allowing the user to interact with the visualization, since optimizing the visualization a priori for all inference tasks simultaneously is not possible, much like creating a single plot for all numerical inference tasks is not possible.

\subsection{Psychophysical Analyses}

The quintessential studies on how a wordcloud is interpreted by humans can be found in (Rivadeneira et al., 2007) and (Bateman et al., 2008). They both investigated various measures of impression-forming and recall to determine which aesthetics conveyed information most effectively - font size chief among them.

Rivandeneira et al. (Rivadeneira et al., 2007) also found that word-order was important for impression forming (displaying words from most frequent to least frequent was most effective here), while displaying words alphabetically was best when searching for a known word. They also found that users prefer a search box when searching for something specific and known, and a wordcloud for exploratory tasks and things unknown.

Bateman et al. (Bateman et al., 2008) examined the relative utility of other aesthetics to convey information, finding that font-weight (boldness) and intensity (opacity) are effective, but not as good as font-size. Aesthetics such as color, number of characters or the area covered by the word were less effective.

Significant research has gone in to the placement of words in the wordcloud (e.g., (Seifert et al., 2008)), though seemingly little information can be conveyed by these layouts (Schrammel et al., 2009). Indeed, (Rivadeneira et al., 2007) indicates that words directly adjacent to the largest word in the wordcloud had slightly worse recall than those not-directly-adjacent - in essence, getting the most important words in the center may be counterproductive. Thus we eschew these algorithms in favor of more interpretable (but perhaps less aesthetically pleasing) linear ordered layouts.

\subsection{Wordclouds as a tool}

Illustrative investigations of the wordcloud as a tool for exploratory data analysis are few, but encouraging.

In relation to question (1), even static wordclouds can be useful for this task. Users performing an open-ended search task preferred using a wordcloud to a search box (Sinclair and Cardew-Hall, 2008), possibly because the wordcloud prevented them from having to hypothesize what might be in the collection before searching for it. Similarly, wordclouds can be used as a follow-up display of search results from a query performed via a standard text search box (Knautz et al., 2010), providing the user a crude summary of the results. In both of these cases, a simple static wordcloud is able to provide some useful information to the user, though less research has been done to determine the optimal composition of the wordcloud. What's more, the need for a dynamic interactive wordcloud was made explicit (Knautz et al., 2010), given the way the users iteratively refined their queries and wordclouds.

Question (2) has also been examined. One approach is to make a set of wordclouds with soft constraints that the same word appears in roughly the same position across multiple clouds to facilitate comparisons (Castella and Sutton, 2013). Each of these clouds in a wordstorm visualizes a different collection of documents (e.g., subdivisions via metadata of a larger corpus).

Similarly addressing our second question, Parallel Tag Clouds (Collins et al., 2009) allow the comparison of multiple sets of documents (or different partitions of a corpus). This investigation provides a theoretically-justified approach to finding 'the right' static wordcloud (for a single inference task), though this does depend on some language-specific resources (e.g., stopword lists and stemming). Interestingly, they opt for ex- 
plicit removal of words and outliers that the user does not wish to have displayed (an exclusion list), rather than adjusting calculations of the entire cloud to remove them in a principled and fair manner.

\subsection{Wordclouds and Metadata}

Wordclouds have previously been extended to convey additional information, though these adaptations have been optimized generally for artistic purposes rather than exploratory data analysis.

Wordclouds can been used to display how language interacts with a temporal dimension in (Dubinko et al., 2007; Cui et al., 2010; Lee et al., 2010). Dubinko and colleagues created a tag cloud variant that displays trends in tag usage over time, coupled with images that have that tag (Dubinko et al., 2007). An information-theoretic approach to displaying information changing in time gives rise to a theoretically grounded approach for displaying pointwise tag clouds, and highlighting those pieces that have changed significantly as compared to a previous time period (Cui et al., 2010). This can be viewed as measuring the change in overall language usage over time. In contrast, using spark lines on each individual word or tag can convey temporal trends for individual words (Lee et al., 2010).

Meanwhile, combining tag clouds with geospatial data yields a visualization where words can be displayed on a map of the world in locations they are frequently tagged in, labeling famous landmarks, for example (Slingsby et al., 2007).

\section{Desiderata}

In light of the diverse inference tasks ( $(2)$ and prior art ( $\$ 5)$, the following desiderata emerge for the visualization. These desiderata are explicit choices, not all of which are ideal for all inference tasks. Thus, chief among them is the first: flexibility to allow maximum extensions and modifications as needed.

Flexible and adjustable in real time: Any single static wordcloud is guaranteed to be suboptimal for at least some inference tasks, so allowing the user to adjust the aspect-to-aesthetic mapping of the wordcloud in real time enables adaptation of the visualization to the data and inference task at hand. The statistics described in $\S 4$ are relevant to every language collection (and most inference tasks), yet there are a number of other ordinal val- ues to associate a word (e.g., the weight assigned to it by a classifier). Thus, $t f$ and $i d f$ are meant to be illustrative examples though the visualization code should generalize well to others.

Though removal of the most frequent words (stopwords) is useful in many natural language processing tasks, there are many ways to define which words fall under this category. Unsurprisingly, the optimal selection of these words can also depend upon the task at hand (e.g., psychiatric v. thematic analysis as in $\S 2$ ), so maximum flexibility and minimum latency are desirable.

Interpretable: An explicit legend is needed to interpret the differences in visual aesthetics and what these differences mean with respect to the underlying data aspects.

Language-Agnostic: We need methods for exploratory data analysis that work well regardless of the language(s) being investigated. This is crucial for multilingual corpora, yet decidedly nontrivial. These techniques must be maximally language-agnostic, relying on only the most rudimentary understanding of the linguistic structure of the data (e.g., spaces separate words in English, but not in Chinese), so they can be extended to many languages easily.

This precludes the use of a fixed set of stop words for each language examined, since a new set of stopwords would be required for each language explored. Alternatively, the set of stopwords can be dealt with automatically, either by granting the user the ability to filter out words in the extremes of the distributions ( $t f$ and $d f$ alike) through the use of a weight which penalizes these ubiquitous or too-rare words. Similarly precluded is the use of stemming to deal with the many surface forms of a given root word (e.g., type, typing, typed).

\section{Dynamic Wordclouds}

We address Question (1) and a number of our desiderata with the addition of explicitly labeled controls to the static wordcloud display, which allows the user to control the mapping from data aspects to the visualization aesthetics. We supplement these controls with an explicit explanation of how each aesthetic is affected by each aspect, so the user can easily read the relevant mappings, rather than trying to interpret the location of the sliders. An example of which is that "Larger words are those that frequently occur in the query", when the aspect $t f$ is mapped to the 


\begin{tabular}{|c|c|c|}
\hline 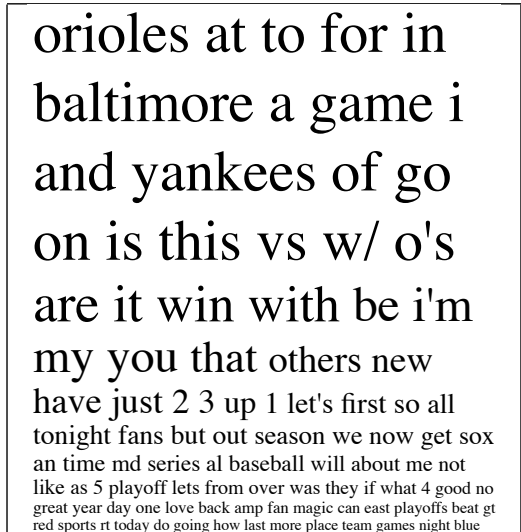 & 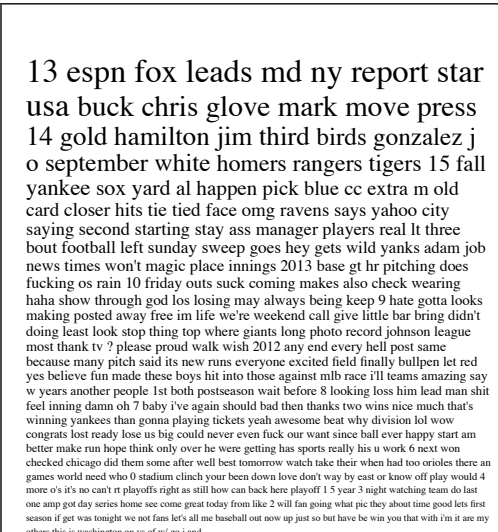 & 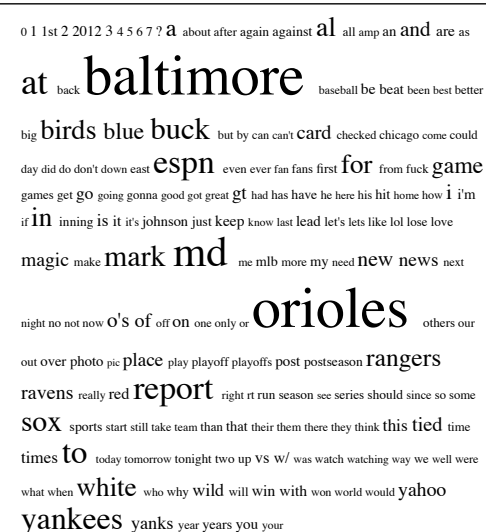 \\
\hline
\end{tabular}

Figure 1: Three example settings of the dynamic wordcloud for the same set of tweets containing "Orioles". Left: size reflects $t f$, sorted by $t f$; Center: size reflects $i d f$, sorted by $i d f$; Right: size reflects $t f * i d f$, sorted alphabetically.

aesthetic font-size (and this description is tied to the appropriate sliders so it updates as the sliders are changed). The manipulation of the visualization in real time allows us to take advantage of the human's adept visual change-detection to highlight and convey the differences between settings (or a range of settings), even subtle ones.

The data aspects from $\S 4$ are precomputed and mapped to the aesthetics from $\S 3$ in a JavaScript visualization displayed in a standard web browser. This visualization enables the user to manipulate the aspect-to-aesthetic mapping via an intuitive set of sliders and buttons, responsive in real time. The sliders are roughly segmented into three categories: those that control which words are displayed, those that control how size is calculated, and those that control how opacity is calculated. The buttons control the order in which words appear.

One set of sliders controls which words are displayed by examining the frequency and rarity of the words. We define the range $\tau_{\text {Freq }}=$ $\left[t_{\text {Freq }}^{\text {min }}, t_{\text {Freq }}^{\text {max }}\right]$ as the range of $t f$ values for words to be displayed (i.e., $t f(w) \in \tau_{\text {Freq }}$ ). The viewer is granted a range slider to manipulate both $t_{F r e q}^{\text {min }}$ and $t_{F r e q}^{\max }$ to eliminate words from the extremes of the distribution. Similarly for $d f$ and $\tau_{\text {Rarity }}$. Those words that fall outside $\tau_{F r e q}$ or $\tau_{\text {Rarity }}$ are not displayed. Importantly, $t f$ is computed from the current corpus displayed while $d f$ is computed over a much larger collection (in our running examples, all the works of Shakespeare or all the tweets for the last 6 months). Those with high $d f$ or high $t f$ are often stopwords, those with low $t f$ and low $d f$ are often rare, sometimes too rare to get good estimates of $t f$ or $i d f$ (e.g., names).
A second set of sliders controls the mapping between aspects and aesthetics for each individual word. Each aesthetic has a weight for the importance of rarity $\left(\gamma_{\text {Rarity }}\right)$ and the importance of frequency $\left(\gamma_{F r e q}\right)$, corresponding to the current values of their respective slider (each in the range $[0,1])$. For size, we compute a weight attributed to each data aspect:

$$
\omega_{F r e q}(w)=\left(1-\gamma_{F r e q}\right)+\gamma_{F r e q} t f(w)
$$

and similarly for Rarity.

In both cases, the aesthetic's value is computed via an equation similar to the following:

$$
a(w)=\omega_{\text {Freq }}(w) \omega_{\text {Rarity }}(w) \gamma_{\text {Range }} b
$$

where $a(w)$ is either font size or opacity, and $b$ is some base value of the aesthetic (scaled by a dynamic range slider, $\gamma_{\text {Range }}$ ) and the weights for frequency and rarity of the word. In this manner, the weights are multiplicative, so interactions between the variables (e.g., $t f^{*} i d f$ ) are apparent.

Though unigram statistics are informative, seeing the unigrams in context is also important for many inference tasks. To enable this, we use reservoir sampling (Vitter, 1985) to maintain a representative sample of the observed occurrences of each word in context, which the user can view by clicking on the word in the wordcloud display.

Examples of the dynamic wordcloud in various settings can be found in Figure 1, using a set of tweets containing "Orioles". The left wordcloud has $t f$ mapped to size, the center with $i d f$ mapped to size, and the right with both high $t f$ and high $i d f$ mapped to size. We only manipulate the size aesthetic, since the opacity aesthetic is sometimes hard to interpret in print. To fit the wordclouds 
to the small format, various values for $\tau_{F r e q}$ and $\tau_{\text {Rarity }}$ are employed, and order is varied - the left is ordered in descending order in terms of frequency, the center is ordered in descending order in terms of rarity, and the right is in alphabetical order.

\section{Vennclouds}

Question (2) - "how are these corpora related" requires only a single change to the dynamic single wordcloud described in $\S 7$. We refer to two corpora, left and right, which we abbreviate $L$ and $R$ (perhaps a set of tweets containing "Orioles" for left and those containing "Nationals" for right as in Figure 2). For the right documents, let $R=\left\{d_{1}, \ldots, d_{n_{R}}\right\}$ so $|R|=n_{R}$ and let $T_{R}$ be the total number of tokens in all the documents in $R$

$$
T_{R}=\sum_{d \in R}\left|T_{d}\right|
$$

We separate the wordcloud display into three regions, one devoted to words most closely associated with $R$, one devoted to words most closely associated with $L$, and one for words that should be associated with both. "Association" here can be defined in a number of ways, but for the nonce we define it as the probability of occurrence in that corpus - essentially term frequency, normalized by corpus length. Normalizing by length is required to prevent bias incurred when the corpora are different sizes $\left(T_{L} \neq T_{R}\right)$. Specifically, we define the number of times $w$ occurs in left $(t f)$ as

$$
t f_{L}(w)=\sum_{d_{i} \in L} T\left(w, d_{i}\right)
$$

and this quantity normalized by the number of tokens in $L$,

$$
\overline{t f_{L}}(w)=t f_{L}(w) / T_{L}
$$

and this quantity as it relates to the term frequency of this $w$ in both corpora

$$
\overline{t f_{L \mid R}}(w)=\frac{t f_{L}(w)}{t f_{L}(w)+t f_{R}(w)}
$$

Each word is only displayed once in the Venncloud (see Figure 2, so if a word $(w)$ only occurs in $R$, it is always present in the right region, and likewise for $L$ and left. If $w$ is in both $L$ and $R$, we examine the proportion of documents in each that $w$ is in and use this to determine in which region it should be displayed. In order to deal with

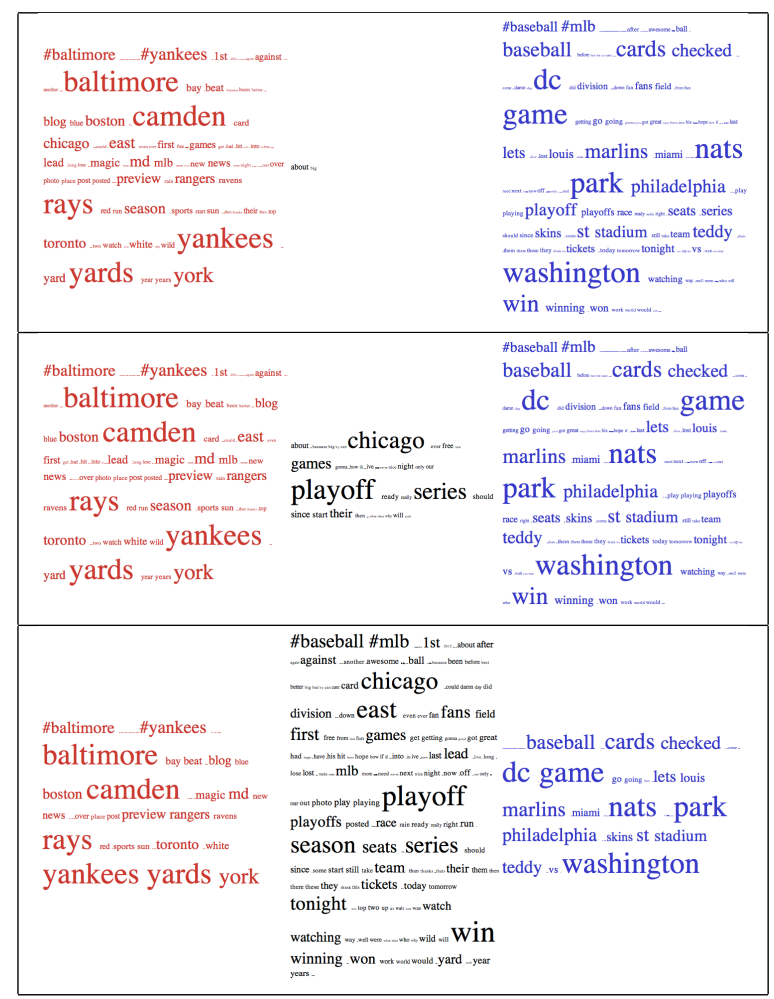

Figure 2: Three example Vennclouds, with tweets containing "Orioles" on the left, "Nationals" on the right, and common words in the middle. From top to bottom we allow progressively larger common clouds. The large common words make sense - both teams played a Chicago team and made the playoffs in the time covered by these corpora.

the cases where $w$ occurs in approximately similar proportions of left and right documents, we have a center region (in the center in Figure 2). We define a threshold $\left(\tau_{\text {Common }}\right)$ to concretely define "approximately similar". Specifically,

- if $t f_{R}(w)=0, w$ is displayed in left.

- if $t f_{L}(w)=0, w$ is displayed in right.

- if $t f_{R}(w)>0$ and $t f_{L}(w)>0$,

- if $\overline{t f_{R \mid L}}(w)>\overline{t f_{L \mid R}}(w)+\tau_{\text {Common }}, w$ is displayed in right.

- if $\overline{t f_{L \mid R}}(w)>\overline{t f_{R \mid L}}(w)+\tau_{\text {Common }}, w$ is displayed in left.

- Otherwise, $w$ is displayed in center.

The user is given a slider to control $\tau_{\text {Common, al- }}$ lowing them to determine what value of "approximately similar" best fits the data and their task at hand.

\section{Anecdotal Evaluation}

We have not yet done a proper psychophysical evaluation of the utility of dynamic wordclouds 


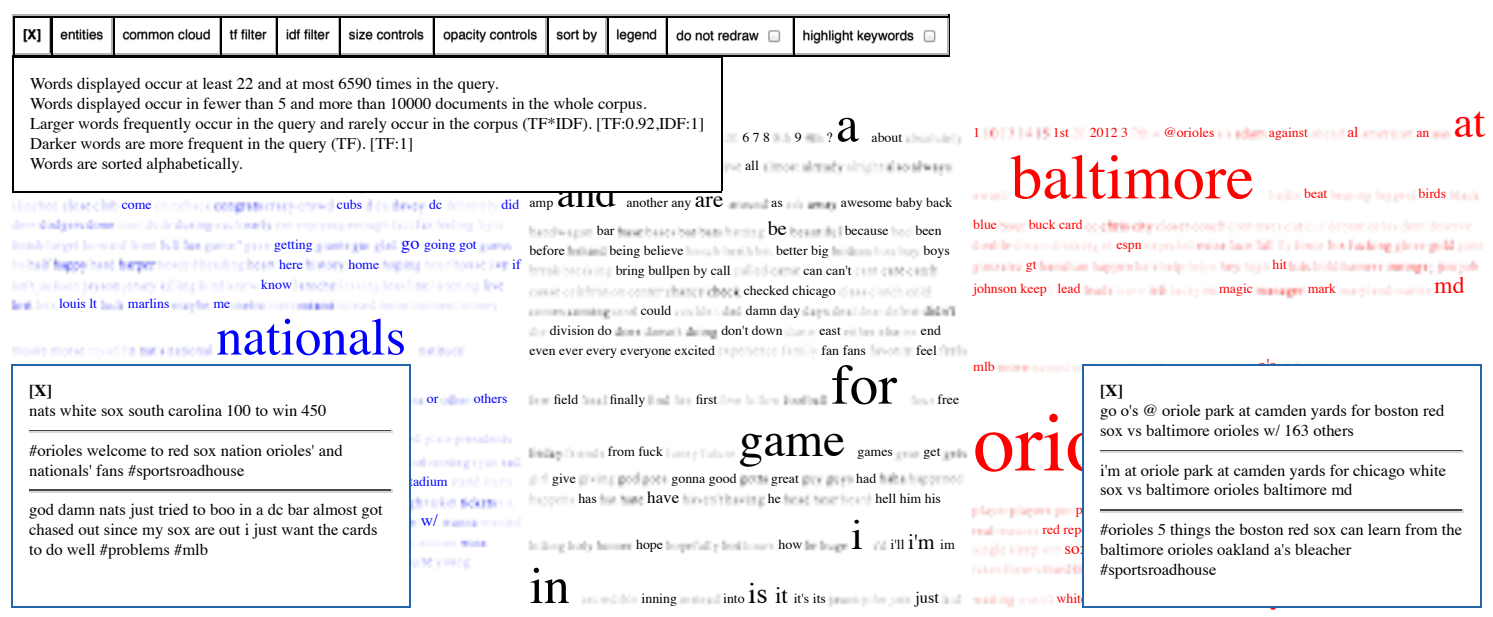

Figure 3: Screenshot of a Venncloud, with controls. The sliders are accessible from the buttons across the top, displaying as a floating window above the wordcloud itself (replacing the current display of the legend). Also note the examples in the lower left and right corners, accessed by clicking on a word of interest (in this case "Sox").

and Vennclouds for various tasks as compared to their static counterparts (and other visualizations). In part, this is because such an evaluation requires selection of inference tasks to be examined, precisely what we do not claim to be able to do. We leave for future work the creation and evaluation of a representative sample of such inference tasks.

We strongly believe that the plural of anecdote is not data - so these anecdotes are intended as illustrations of use, rather than some data regarding utility. The dynamic wordclouds and Vennclouds were used on data from across the spectrum, from tweets to Shakespeare and political speeches to health-related conversations in developing nations. In Shakespeare, character and place names can easily be highlighted with one set of slider settings (high $t f * i d f$ ), while comparisons of stopwords are made apparent with another (high $t f$, no $i d f$ ). Emerging from the debates between Mitt Romney and Barack Obama are the common themes that they discuss using similar (economics) and dissimilar language (Obama talks about the "affordable care act" and Romney calls it "Obamacare"). These wordclouds were also used to do some introspection on the output of classifiers in sentiment analysis (Mitchell et al., 2013) and mental health research (Coppersmith et al., 2014b) to expose the linguistic signals that give rise to successful (and unsuccessful) classification.

\section{Conclusions and Future Directions}

Exploratory data analysis tools for human language data and inference tasks have long lagged behind their numerical counterparts, and here we investigate another step towards filling that need. Rather than determining the optimal wordcloud, we enable the wordcloud viewer to adapt the visualization to the data and inference task at hand. We suspect that the pendulum of control has swung too far, and that there is a subset of the possible control configurations that produce useful and informative wordclouds. Work is underway to collect feedback via instrumented dynamic wordclouds and Vennclouds as they are used for various inference tasks to address this.

Previous research, logic, and intuition were used to create this step, though it requires further improvement and validation. We provide anecdotes about the usefulness of these dynamic wordclouds, but those anecdotes do not provide sufficient evidence that this method is somehow more efficient (in terms of human time) than existing methods. To make such claims, a controlled human-factors study is required, investigating (for a particular inference task) how this method affects the job of an exploratory data analyst. In the meantime, we hope making the code freely available ${ }^{1}$ will better enable our fellow researchers to perform principled exploratory data analysis of human language content quickly and encourage a deeper understanding of data, within and across disciplines.

\section{Acknowledgments}

We would like to thank Carey Priebe for insightful discussions on exploratory data analysis,

\footnotetext{
${ }^{1}$ from https://github.com/Coppersmith/vennclouds
} 
Aleksander Yelskiy, Jacqueline Aguilar, Kristy Hollingshead for their analysis, comments, and improvements on early versions, and Ainsley R. Coppersmith for permitting this research to progress in her early months.

\section{References}

Scott Bateman, Carl Gutwin, and Miguel Nacenta. 2008. Seeing things in the clouds: the effect of visual features on tag cloud selections. In Proceedings of the nineteenth ACM conference on Hypertext and hypermedia, pages 193-202. ACM.

Quim Castella and Charles A. Sutton. 2013. Word storms: Multiples of word clouds for visual comparison of documents. CoRR, abs/1301.0503.

Cindy Chung and James W Pennebaker. 2007. The psychological functions of function words. Social communication, pages 343-359.

Christopher Collins, Fernanda B Viegas, and Martin Wattenberg. 2009. Parallel tag clouds to explore and analyze faceted text corpora. In Visual Analytics Science and Technology, 2009. VAST 2009. IEEE Symposium on, pages 91-98. IEEE.

Glen Coppersmith, Mark Dredze, and Craig Harman. 2014a. Quantifying mental health signals in twitter. In Proceedings of ACL Workshop on Computational Linguistics and Clinical Psychology. Association for Computational Linguistics.

Glen Coppersmith, Craig Harman, and Mark Dredze. 2014b. Measuring post traumatic stress disorder in Twitter. In Proceedings of the International AAAI Conference on Weblogs and Social Media (ICWSM).

Weiwei Cui, Yingcai Wu, Shixia Liu, Furu Wei, Michelle X Zhou, and Huamin Qu. 2010. Context preserving dynamic word cloud visualization. In Pacific Visualization Symposium (PacificVis), 2010 IEEE, pages 121-128. IEEE.

Jason Davies. 2013. Wordcloud generator using d3, April.

Micah Dubinko, Ravi Kumar, Joseph Magnani, Jasmine Novak, Prabhakar Raghavan, and Andrew Tomkins. 2007. Visualizing tags over time. ACM Transactions on the Web (TWEB), 1(2):7.

Jason Feinberg. 2013. Wordle, April.

Patrick Juola. 2006. Authorship attribution. Foundations and Trends in information Retrieval, 1(3):233334.

Kathrin Knautz, Simone Soubusta, and Wolfgang G Stock. 2010. Tag clusters as information retrieval interfaces. In System Sciences (HICSS), 2010 43rd Hawaii International Conference on, pages 1-10. IEEE.
Bongshin Lee, Nathalie Henry Riche, Amy K Karlson, and Sheelagh Carpendale. 2010. Sparkclouds: Visualizing trends in tag clouds. Visualization and Computer Graphics, IEEE Transactions on, 16(6):1182-1189.

Margaret Mitchell, Jacqueline Aguilar, Theresa Wilson, and Benjamin Van Durme. 2013. Open domain targeted sentiment. In Proceedings of the 2013 Conference on Empirical Methods in Natural Language Processing, pages 1643-1654. Association for Computational Linguistics.

Saša Petrovic, Miles Osborne, Richard McCreadie, Craig Macdonald, Iadh Ounis, and Luke Shrimpton. 2013. Can twitter replace newswire for breaking news. In Seventh International AAAI Conference on Weblogs and Social Media.

Paul Rayson and Roger Garside. 2000. Comparing corpora using frequency profiling. In Proceedings of the workshop on Comparing Corpora, pages 1-6. Association for Computational Linguistics.

AW Rivadeneira, Daniel M Gruen, Michael J Muller, and David R Millen. 2007. Getting our head in the clouds: toward evaluation studies of tagclouds. In Proceedings of the SIGCHI conference on Human factors in computing systems, pages 995-998. ACM.

Johann Schrammel, Michael Leitner, and Manfred Tscheligi. 2009. Semantically structured tag clouds: an empirical evaluation of clustered presentation approaches. In Proceedings of the 27th international conference on Human factors in computing systems, pages 2037-2040. ACM.

Christin Seifert, Barbara Kump, Wolfgang Kienreich, Gisela Granitzer, and Michael Granitzer. 2008. On the beauty and usability of tag clouds. In Information Visualisation, 2008. IV'08. 12th International Conference, pages 17-25. IEEE.

James Sinclair and Michael Cardew-Hall. 2008. The folksonomy tag cloud: when is it useful? Journal of Information Science, 34(1):15-29.

Aidan Slingsby, Jason Dykes, Jo Wood, and Keith Clarke. 2007. Interactive tag maps and tag clouds for the multiscale exploration of large spatiotemporal datasets. In Information Visualization, 2007. IV'07. 11th International Conference, pages 497-504. IEEE.

Fernanda B Viégas and Martin Wattenberg. 2008. Timelines tag clouds and the case for vernacular visualization. interactions, 15(4):49-52.

Jeffrey S Vitter. 1985. Random sampling with a reservoir. ACM Transactions on Mathematical Software (TOMS), 11(1):37-57.

Hadley Wickham. 2009. ggplot2: elegant graphics for data analysis. Springer Publishing Company, Incorporated. 